\title{
Epidemic transmission on SEIR stochastic models with nonlinear incidence rate
}

\author{
M.J. Lopez-Herrero \\ Lherrero@ucm.es \\ Faculty of Statistical Studies, Complutense University of Madrid \\ 28040 Madrid
}

Our interest is to quantify the spread of an infective process with latency period and generic incidence rate that takes place in a finite and homogeneous population.

Within a stochastic framework, two random variables are defined to describe the variations of the number of secondary cases produced by an index case inside of a closed population. Computational algorithms are presented in order to characterize both random variables. Finally, theoretical and algorithmic results are illustrated by several numerical examples.

Keywords: Nonlinear incidence rate; Stochastic SEIR epidemic model; Index case; Secondary cases.

\section{Introduction}

A very large number of epidemiological models involves a compartmental division of individuals. It is also commonly assumed that individuals make contacts at random showing no preferences. One of the fundamental compartmental models is the SIR proposed by Kermack \& McKendrick [1], where individuals are classified as susceptible to the infection, labeled $S$, infected by the pathogen, $I$, or recovered from the infection, $R$. There are many hypothesis behind this model, which refer to population's size (a large one, a closed one, with or without natural births and deaths during the outbreaks), the lack of a latency period (individuals become infectious as soon as they become infected), lifetime immunity after recovery, and homogeneous mixing.

This paper regards the study of the spread of a communicable disease involving latency period and a general mode of transmission so, we generalize the classic SIR model in two ways. First we deal with an SEIR model, that is, we split the infected population group into two ones: latent or in exposed period individuals and infectious individuals able to spread the infection. Second, we consider a transmission of nonlinear type.

The mode of transmission determines the possible response of the disease upon study. Its mathematical description is based in a function called force 
of infection or incidence rate. In most models transmission is assumed to occur via so called mass action [2], involving a bilinear function of $S$ and $I$. This particular choice makes that theoretical models show an exponential growth in the early part of the epidemics ([1], [2]).

Bilinear incidence rate is a natural assumption in accordance with the homogeneous mixing hypothesis but it is dubious upon heterogeneous. In that sense, recent studies ([3], [4]), based on empirical infection diseases datasets, revealed that a slower than exponential initial growth is observed in populations with highly constrained contact structures that are suffering from infectious diseases transmitted via close contacts (e.g. Ebola, SARS, STDs, etc.). These works provide an evidence that considering nonlinear transmission rates seems a more realistic assumption than linearity, specially with regard to recover sub-exponential growth profiles. Some authors ([5], [6]) generalize the bilinear rate to a power function, $I^{p} S^{q}$, or introduce a deviation from the bilinear choice by using a rate of the form $I S(1+\alpha I$ ) (see [7] and the references therein). Korobeinikov \& Maini [8] consider nonlinear transmission to incorporate the population structure and heterogeneous mixing into the model. More precisely, they consider an increasing function of $S$ that is also increasing and concave respect to the number of infective individuals. Zhang et al. [9] and Lahrouz \& Omari [10] consider a transmission rate of the form $I S / \psi(I)$, with $\psi(I)$ positive and non decreasing; which covers well-known incidence functions such as the proposed by Capasso \& Serio [11] arising from saturation effects $(\psi(I)=1+\alpha I)$, or the one reflecting psychological effects $\left(\psi(I)=1+\alpha I^{2}\right)$ proposed Xiao \& Ruan [12]. More recently, Li et al. [13] choose a general incidence rate of the form $S G(I)$, with $G(I)$ not necessarily increasing, which includes the mass action, saturated incidence and incidence with media coverage ([14], [15]).

More examples of nonlinear infection rates and their effect on the population can be found in the works by Hethcote \& Levin [16], Alexander \& Moghadas [17] and Turnes \& Monteiro [18].

Measuring the transmission potential in a population is the role of $R_{0}$, the basic reproduction number (see [19] or [20]). This is the most widely used measure of disease spread in epidemiology and population dynamics. $R_{0}$ is a threshold parameter which tries to measure the initial spread of an epidemic process by estimating the average of secondary cases of infection arising from an index case in a virgin population, during its infectious period. Artalejo \& Lopez-Herrrero include in [21] a summary of remarkable features and drawbacks of $R_{0}$, which is supported by an important set of references. It is not the aim of the author to repeat same arguments here so, we kindly address the interested reader to [21] and the references therein. 
In the stochastic framework it is frequently assumed a value for $R_{0}$ inherited from the deterministic counterpart. In that sense, for our stochastic SEIR model with non-linear incidence rate, we call basic reproduction number to the quantity appearing at expression (3.7) in the paper by Korobeinikov \& Maini [8], determined by van den Driessche \& Watmough [22] using the next generation method, whenever the non-linear incidence rate satisfies conditions stated on [22]. We remark that, for a deterministic model without vital dynamics, the expression for $R_{0}$, is the same in SIR and SEIR models; showing that the average number of individuals infected by an index case does not depend on the exposed population. And one wonders if this coincidence is not a consequence of estimating an average number in a large population.

When dealing with a small population, where the stochastic approach is preferable [23], the validity of the linearization hypothesis assumed in a deterministic framework is questionable. To neglect the impact of depletion of susceptible individuals due to the infective process produces an overestimation on the number of secondary cases which correspond to contacts between the index case and individuals previously infected.

In the paper we present two measures alternative to $R_{0}$ focusing on a stochastic perspective. For an epidemic process described by a continuous time Markov chain $(C T M C)$ we quantify the steady-state behavior of the epidemic spread by means of two random variables. Namely, $R_{e 0}$ : the exact reproduction number and $R_{p}$ or the population transmission number. The aim of this paper is two fold:

- To extent the existing methodology to a more involved epidemic model and to find stable computational schemes able to handle the high dimensionality of the state spaces arising when an additional component (i.e., latency period) is introduced in the Markovian model representing the epidemic evolution within the population.

- To study the influence of the latency parameter to the epidemic spread in an SEIR model, when transmission depends on a general nonlinear incidence function. In particular we investigate whether $R_{e 0}$ and $R_{p}$ distributions depend or not on latency rate for a specific group of incidence functions including the usual bilinear mass action.

The organization of the rest of the paper is as follows. In Section 2, we introduce the mathematical description of the stochastic SEIR model as a continuous time Markov chain. In Sections 3 and 4, starting from a fixed initial state, we define above variables and provide algorithmic recursive 
schemes for the computation of the generating functions, mass probability functions and factorial moments of $R_{e 0}$ and $R_{p}$, respectively. Section 5 illustrates numerically the behavior of both random variables. Last, in Section 6 , we discuss our results.

\section{Model description}

Consider a closed population of $\mathrm{N}$ individuals which is affected by a contagious disease conferring immunity and having a latency period between being infected and becoming infective. At any time, individuals in the population are classified into four homogeneous compartments according to his disease state. More specifically, at a given point time $t$ we denote by $S(t)$ the number of susceptible individuals, by $E(t)$ the number of exposed or individuals in latent period, by $I(t)$ the number of infectious individuals and by $R(t)$ the number of recovered or immune individuals.

Latency periods are assumed to be independent and identical distributed with length described by an exponential law having a progression rate $\sigma$. Each individual recovers independently of the rest of infectious individuals according to an exponential law with rate $\gamma$. Disease transmission is produced through direct contact between an infective individual and a susceptible; these contact occur at the time points of a time homogeneous Poisson process; infection rate will depend on the number of infective and susceptible through a finite incidence function $\beta(s, i) \equiv \beta_{s i}$. After recovery, individuals play no role in the epidemic spread; consequently the epidemic stops as soon as the population contains no infected (i.e., exposed or infectious) individuals. In addition, all the involved Poisson processes are assumed to be independent.

As we are dealing with a closed population, there is no need of recording all compartment's occupancy level. So, the number of recovered individuals is not reported and the stochastic SEIR model is a three dimensional continuous time Markov chain $X=\{(S(t), E(t), I(t)): t>0\}$ that provides the number of susceptible, exposed and infectious individuals at any time $t$ and whose state space is $S=S_{1}-S_{2}$ with $S_{1}=\{(s, e, i): 0 \leq$ $s, e, i \leq N, s+e+i \leq N\}$ and $S_{2}=\{(s, e, 0): 0 \leq s, e \leq N, s+e=N\}$. Notice that $S$ is a finite set having $|S|=N(N+1)(N+5) / 6$ states in. We assume that any epidemic outbreak starts with a single infectious individual in a totally susceptible population, hence the initial state of $X$ is $(S(0), E(0), I(0))=(N-1,0,1)$. If we order the states in $S$ according to natural order, that is, $S=\{(s, e, i): 0 \leq s \leq N-1,0 \leq e \leq N-s-1,0 \leq$ 
$i \leq N-s-e\}$, we notice that a state $(s, e, i)$ is accessible from another $\left(s^{\prime}, e^{\prime}, i^{\prime}\right)$ when $(s, e, i) \leq\left(s^{\prime}, e^{\prime}, i^{\prime}\right)$ respect to the natural order above. Moreover, we observe that once the CTMC $X$ departs from a state never returns to it. In long terms, the process enters into the set of absorbing states $S_{A}=\{(s, 0,0): 0 \leq s \leq N-1\}$ which correspond to the end of the infectious disease within the closed population. Due to the reducible character of $X$, the stationary distribution becomes degenerate in the sense that it only assingns probability mass to states in $S_{A}$, which guarantees the certain extinction of the epidemic process.

Soujourn times at each state $(s, e, i)$ are independent exponential random variables with rates determined by means of the infinitesimal generator $Q$, whose entries are described as follows

$$
q_{(s, e, i)\left(s^{\prime}, e^{\prime}, i^{\prime}\right)}=\left\{\begin{array}{lll}
\beta_{s i}, & \text { if } & \left(s^{\prime}, e^{\prime}, i^{\prime}\right)=(s-1, e+1, i), \\
\sigma e, & \text { if } & \left(s^{\prime}, e^{\prime}, i^{\prime}\right)=(s, e-1, i+1), \\
\gamma i, & \text { if } & \left(s^{\prime}, e^{\prime}, i^{\prime}\right)=(s, e, i-1), \\
-q_{s e i}, & \text { if } & \left(s^{\prime}, e^{\prime}, i^{\prime}\right)=(s, e, i), \\
0, & & \text { otherwise. }
\end{array}\right.
$$

where $q_{s e i}=\beta_{s i}+\sigma e+\gamma i$, for $(s, e, i) \in S$.

Matrix $Q$ can be expressed in a block lower triangular form by partitioning the state space in level states: $S=\cup_{s=0}^{N-1} l_{s}$, where $l_{s}$ is the $s$-th level that contains states with exactly $s$ susceptible individuals. Each level state is partitioned, in turns, in sub-levels according to the number of exposed individuals, that is $l_{s}=\cup_{e=0}^{N-s-1} l_{s e}$. Details can be found in [24]. This partition will be useful when searching for numerical solutions of systems of equations trough recursive computationally stable schemes.

\section{The exact reproduction number $R_{e 0}$}

The distribution of the offspring distribution of secondary infections at a particular stage of an epidemic assuming Markov chain compartmental models was focused first by Ross in [25] and, in an independent way, by Artalejo and Lopez-Herrero in [21]. Both papers evaluate the probability mass function of secondary infections by iteratively solving a set of linear equations. Methodology in [25] is applied to phase type infectious periods, producing efficient results for small populations but the size of the matrices involved are extremely large and computational costs prevent the extension of this methodology to more complicated models. On the other hand, methodology given in [21] involves first-step arguments and solves systems of linear 
equations using a stable iterative procedure that avoids the use and storage of large matrices, which permits to handle populations of up to 10,000 individuals, when dealing with classical SIS and SIR models.

Our aim is to extend the underlying methodological ideas to characterize the distribution of the exact reproduction number, $R_{e 0}$, for the Markov chain SEIR compartmental model and observe the influence of long latent periods on the epidemic spread.

In this Section we present stable algorithmic schemes for determining the distribution of $R_{e 0}$, via generating function, mass probability function and moments. Numerical experiments in Section 5, obtained by implementing their corresponding computational algorithms, will show that the distribution of the exact reproduction number depends upon the latency time even when the mass action transmission function is considered, in contradistinction to what happens in deterministic SIR and SEIR models without demographic parameters.

Let us begin by introducing the random variable $R_{e 0}$ associated to the infection expansion which is defined as follows:

$R_{e 0}$ is the exact number of secondary cases arising from a single infective individual during its entire infectious period.

We notice that this random variable is defined to correct the effect of the linearization assumption because vain contacts (i.e., those contacts occurring between the selected infective and any contacted individual previously infected) are ignored. In particular, the basic reproduction number, $R_{0}$, is defined as the expected value of $R_{e 0}$ conditioned to the initial state $(N-1,0,1)$ for the process $X$, that is a single infective in a virgin population. As $R_{e 0}$ is a random variable we can get more information about it than an expected value under a fixed circumstance, so in what follows we proceed by studying the whole distribution (via generating and mass probability functions) and also its moments.

We start by introducing an appropriate notation for the generating and probability mass functions of $R_{e 0}$, and factorial moments conditioned to the current situation.

$$
\begin{aligned}
\varphi_{s e i}(z) & =\sum_{n=0}^{s} z^{n} P\left\{R_{e 0}=n \mid(S(0), E(0), I(0))=(s, e, i)\right\}, \text { for }|z| \leq 1, \\
x_{\text {sei }}^{n} & =P\left\{R_{e 0}=n \mid(S(0), E(0), I(0))=(s, e, i)\right\}, \text { for } 0 \leq n \leq s, \\
m_{\text {sei }}^{l} & =\left\{\begin{array}{l}
0, \text { if } l=0, \\
E\left[R_{e 0}\left(R_{e 0}-1\right) \ldots\left(R_{e 0}-l+1\right)\right], \text { if } l>0,
\end{array}\right.
\end{aligned}
$$

with $(s, e, i) \in S_{T}=S-S_{A}$. 
In order to develop algorithmic schemes for determining $\varphi_{\text {sei }}(z), x_{\text {sei }}^{n}$ and $m_{s e i}^{l}$ we begin by marking the infective individual starting the infective process, next we split the transmission process by distinguishing whether a contact involves or not the initial marked infective individual. In consequence, transmission rate is decomposed as

$$
\beta_{s i}=\beta_{s i}^{*}+\widetilde{\beta}_{s i} \text {, for }(s, e, i) \in S_{T} \text { and } i \geq 1,
$$

where $\beta_{s i}^{*}=\beta_{s i} / i$ and $\widetilde{\beta}_{s i}=(i-1) \beta_{s i} / i$.

By appealing to a first step argument, we find that for a fixed state $(s, e, i) \in S_{T}$ the next transition from that state of the Markov chain is associated to one of the following events: (i) an effective contact between the tagged infective and any susceptible individual, (ii) an effective contact between a non- tagged infective and any susceptible individual, (iii) the end latency period for any exposed individual, (iv) the recovery of a nontagged infective and $(\mathrm{v})$ the recovery of the marked infective individual. The following stable algorithm provides the generating function of the number of effective contacts produced by the tagged infective during its infectious time, from the current state $(s, e, i)$. Notation $\delta_{x y}$ stands for Kronecker's delta, defined as one for $x=y$ and zero otherwise.

Algorithm 1 The conditional generating function $\varphi_{\text {sei }}(z)$ of the number of secondary cases produced by a typical infective given an a current state $(s, e, i) \in S_{T}$ is computed with the help of the following scheme:

Step 1a: Set $s=0$,

Step 1b: Set $e=0$,

Step 1c: Set $i=1$

Step 1d: Compute

$$
\begin{aligned}
\varphi_{s e i}(z) & =\left(1-\delta_{s 0}\right) \frac{\beta_{s i}^{*}}{\beta_{s i}+\sigma e+\gamma i} z \varphi_{s-1, e+1, i}(z) \\
& +\left(1-\delta_{s 0}\right)\left(1-\delta_{i 1}\right) \frac{\widetilde{\beta}_{s i}}{\beta_{s i}+\sigma e+\gamma i} \varphi_{s-1, e+1, i}(z) \\
& +\left(1-\delta_{e 0}\right) \frac{\sigma e}{\beta_{s i}+\sigma e+\gamma i} \varphi_{s, e-1, i+1}(z) \\
& +\left(1-\delta_{i 1}\right) \frac{\gamma(i-1)}{\beta_{s i}+\sigma e+\gamma i} \varphi_{s, e, i-1}(z)+\frac{\gamma}{\beta_{s i}+\sigma e+\gamma i}
\end{aligned}
$$

Step 2a: Set $i=i+1$. If $i \leq N-s-e$, then go to Step $1 d$.

Step 2b: Set $e=e+1$. If $e \leq N-s-1$, then go to Step 1c.

Step 2c: Set $s=s+1$. If $s \leq N-1$, then go to Step $1 b$. 
By differentiating $l \geq 1$ times the equation (1) with regard to $z$ and setting $z=1$, we obtain a new equation that involves conditional moments of $R_{e 0}$, that is, $m_{s e i}^{l}$, for $(s, e, i) \in S_{T}$ are determined from

$$
\begin{aligned}
m_{s e i}^{l} & =\left(1-\delta_{s 0}\right) \frac{\beta_{s i}^{*}}{\beta_{s i}+\sigma e+\gamma i} l m_{s-1, e+1, i}^{l-1} \\
& +\left(1-\delta_{s 0}\right) \frac{\beta_{s i}}{\beta_{s i}+\sigma e+\gamma i} m_{s-1, e+1, i}^{l}+\left(1-\delta_{e 0}\right) \frac{\sigma e}{\beta_{s i}+\sigma e+\gamma i} m_{s, e-1, i+1}^{l} \\
& +\left(1-\delta_{i 1}\right) \frac{\gamma(i-1)}{\beta_{s i}+\sigma e+\gamma i} m_{s, e, i-1}^{l}, i \geq 1 .
\end{aligned}
$$

We notice that for $l=0$ moments satisfy $m_{\text {sei }}^{0}=1$, whenever $(s, e, i) \in$ $S_{T}$ and $i \geq 1$. So, there is a recursive stable algorithm, similar to Algorithm 1 , that provides conditional moments up to any order $l \geq 1$ based on moments of one order less. In more details, we start from order zero moments and substitute expression (1) in Algorithm 1 by equation (2). In particular, we can get numerical values for $\bar{R}_{e 0}=E\left[R_{e 0} \mid(S(0), E(0), I(0))=(N-1,0,1)\right]$ which provides the expected number of secondary cases produced by the single infective individual inserted in a completely susceptible population that is aimed to be registered by the basic reproduction number $R_{0}$.

Algorithm 1 is also the starting point for getting the mass function of $R_{e 0}$, just by using a numerical inversion method (see for instance [26]) applied to generating functions provided by (1). Instead of that, we present a direct method for computing the conditional probability distribution of $R_{e 0}$ given the current state for the CTMC $X$.

A new appeal to the first step analysis methodology, based on the first transition from a state $(s, e, i) \in S_{T}$ gives

$$
\begin{aligned}
x_{0 e i}^{0}= & 1,0 \leq e \leq N-1,1 \leq i \leq N-e, \\
x_{s e i}^{n}= & \left(1-\delta_{s 0}\right)\left(1-\delta_{n 0}\right) \frac{\beta_{s i}^{*}}{\beta_{s i}+\sigma e+\gamma i} x_{s-1, e+1, i}^{n-1} \\
& +\left(1-\delta_{s 0}\right)\left(1-\delta_{i 1}\right)\left(1-\delta_{n s}\right) \frac{\widetilde{\beta}_{s i}}{\beta_{s i}+\sigma e+\gamma i} x_{s-1, e+1, i}^{n} \\
& +\left(1-\delta_{e 0}\right) \frac{\sigma e}{\beta_{s i}+\sigma e+\gamma i} x_{s, e-1, i+1}^{n}+\left(1-\delta_{i 1}\right) \frac{\gamma(i-1)}{\beta_{s i}+\sigma e+\gamma i} x_{s, e, i-1}^{n} \\
& +\delta_{n 0} \frac{\gamma}{\beta_{s i}+\sigma e+\gamma i},
\end{aligned}
$$

which can be recursively solved directly after the procedure described in the next algorithm. 
Algorithm 2 The set of probabilities $x_{\text {sei }}^{n}$ describing the mass function of $R_{e 0}$ conditioned to the current situation $(s, e, i) \in S_{T}$, are determined as follows:

Step 1: Set $n=0$.

Step 2a: Set $s=0$.

Step 2b: Set $e=0$.

Step 2c: Set $i=1$.

Step 2d: Compute $x_{\text {sei }}^{n}$ via equations (3) or (4).

Step 3a: Set $i=i+1$. If $i \leq N-s-e$, go to Step 2d.

Step 3b: Set $e=e+1$. If $e \leq N-s-1$, go to Step 2c.

Step 3c: Set $s=s+1$. If $s \leq N-1$, go to Step $2 b$.

Step 4: Set $n=n+1$. If $n \leq N-1$ go to Step 2a.

\section{The population transmission number $\mathbf{R}_{p}$}

This section focuses on the random variable $R_{p}$, or the population transmission number, which is defined as the exact number of secondary cases produced by all currently infective individuals prior to the first recovery. This random variable was first studied in [21] and, for SIS and SIR epidemics models with classical rates, its distribution characteristics are determined through simple explicit formulas.

Assuming that we are able to identify the infection when first removal occurs, maybe because it is a death case, then we notice that if we interpret a recovery as a removal, the random variable $R_{p}$ is a measure of the epidemic spread before the first detection for the outbreak of the epidemic process.

As we did in the previous section, the objective is to characterize the distribution of the random population transmission number in terms of its generating and mass probability function and also providing its moments. First we introduce some notation.

Let us condition on the current situation, described in terms of the CTMC $X$, for $(s, e, i) \in S_{T}$ and $|z| \leq 1$ function

$$
\psi_{\text {sei }}(z)=\sum_{n=0}^{s} z^{n} P\left\{R_{p}=n \mid(S(0), E(0), I(0))=(s, e, i)\right\}
$$

is the generating function of $R_{p}$. Given an integer $l \geq 0$, the factorial moment of order $l$ conditioned to the current situation is

$M_{s e i}^{l}=\delta_{l 0}+\left(1-\delta_{l 0}\right) E\left[R_{p}\left(R_{p}-1\right) \cdots\left(R_{p}-l+1\right) \mid(S(0), E(0), I(0))=(s, e, i)\right]$. 
Finally, we denote by $y_{\text {sei }}^{n}$ the probability of having $n$ secondary cases prior to first recovery, starting from the situation $(s, e, i) \in S_{T}$, that is

$$
y_{\text {sei }}^{n}=P\left\{R_{p}=n \mid(S(0), E(0), I(0))=(s, e, i)\right\} .
$$

As in the precedent section, it is possible to derive generating, mass probability functions and factorial moments in terms of an algorithmic scheme; where recursive equations are obtained using a first step argument. More specifically, conditioning on the first possible event starting from the state $(s, e, i) \in S_{T}$ we achieve the following set of equations involving the generating functions at $|z| \leq 1$ :

$$
\begin{aligned}
\psi_{0 e i}(z)= & 1,0 \leq e \leq N-1,1 \leq i \leq N-e, \\
\psi_{s e i}(z)= & \left(1-\delta_{s 0}\right)\left(1-\delta_{i 0}\right) \frac{\beta_{s i}}{\beta_{s i}+\sigma e+\gamma i} z \psi_{s-1, e+1, i}(z) \\
& +\left(1-\delta_{e 0}\right) \frac{\sigma e}{\beta_{s i}+\sigma e+\gamma i} \psi_{s, e-1, i+1}(z)+\left(1-\delta_{i 0}\right) \frac{\gamma i}{\beta_{s i}+\sigma e+\gamma i} .
\end{aligned}
$$

We can compute numerical values of $\psi_{s e i}(z)$ in the natural order, that is for $0 \leq s \leq N-1,0 \leq e \leq N-s-1$ and $\delta_{e 0} \leq i \leq N-s-e$.

Taking $l \geq 1$ derivatives on (5) and (6) regarding $z$, and evaluating at $z=1$, we get a new set of linear equations involving conditional factorial moments. That is,

$$
\begin{aligned}
M_{0 e i}^{l}= & 0,0 \leq e \leq N-1,1 \leq i \leq N-e \\
M_{s e i}^{l}= & \left(1-\delta_{s 0}\right)\left(1-\delta_{i 0}\right) \frac{\beta_{s i}}{\beta_{s i}+\sigma e+\gamma i} M_{s-1, e+1, i}^{l-1} \\
& +\left(1-\delta_{s 0}\right)\left(1-\delta_{i 0}\right) \frac{\beta_{s i}}{\beta_{s i}+\sigma e+\gamma i} M_{s-1, e+1, i}^{l} \\
& +\left(1-\delta_{e 0}\right) \frac{\sigma e}{\beta_{s i}+\sigma e+\gamma i} M_{s, e-1, i+1}^{l} .
\end{aligned}
$$

We observe that system of equations (7) - (8) involve conditional moments of one order less, which is an advantage for minimizing computational storage.

A new first step argument drives us to the following set of equations governing probabilities, $y_{\text {sei }}^{n}$ for $0 \leq n \leq s$ and $(s, e, i) \in S_{T}$.

$$
\begin{aligned}
y_{0 e i}^{0}= & 1,0 \leq e \leq N-1,1 \leq i \leq N-e \\
y_{s e i}^{n}= & \left(1-\delta_{s 0}\right)\left(1-\delta_{i 0}\right)\left(1-\delta_{n 0}\right) \frac{\beta_{s i}}{\beta_{s i}+\sigma e+\gamma i} y_{s-1, e+1, i}^{n-1} \\
& +\left(1-\delta_{e 0}\right) \frac{\sigma e}{\beta_{s i}+\sigma e+\gamma i} y_{s, e-1, i+1}^{n}+\left(1-\delta_{i 0}\right) \delta_{n 0} \frac{\gamma i}{\beta_{s i}+\sigma e+\gamma i}
\end{aligned}
$$


This system of equations can be computed for a fixed number of secondary cases, $n$, in the natural order : $n \leq s \leq N-1,0 \leq e \leq N-s-1$ and $\delta_{e 0} \leq i \leq N-s-e$; and from these values we get probabilities involving one more secondary case, $y_{\text {sei }}^{n+1}$, and so on.

Following remark shows that under certain assumptions on the incidence function the population potential transmission is not changed by latent periods. The proof is done with the help of equations (5) and (6), and it relies in a two dimensional inductive argument proceeding in stages according to the natural order on $s$ and $e$ for the states $(s, e, i) \in S_{T}$.

Remark 3 In SEIR models having an infection rate function satisfying $\beta_{s i}=i \beta_{s 1}$, the random variable $R_{p}$ does not depend on $\sigma$. Generating and probability functions are given by the following explicit expressions:

$$
\begin{aligned}
\psi_{s e i}(z) & =\psi_{s 01}(z)=\sum_{k=0}^{s}\left(\prod_{j=s-k+1}^{s} A_{j}\right) B_{s-k} z^{k}, \\
y_{s e i}^{n} & =y_{s 01}^{n}=\left(\prod_{j=s-n+1}^{s} A_{j}\right) B_{s-n}, \text { for } 0<n \leq s .
\end{aligned}
$$

Also explicit expressions can be derived for factorial moments. In particular

$$
\begin{aligned}
& M_{\text {sei }}^{1}=M_{s 01}^{1}=\sum_{k=0}^{s-1}\left(\prod_{j=s-k}^{s} A_{j}\right), \text { for } s \geq 1, \\
& M_{s e i}^{2}=M_{s 01}^{2}=2 \sum_{k=0}^{s-2}(k+1)\left(\prod_{j=s-k-1}^{s} A_{j}\right), \text { for } s \geq 2, \\
& M_{s e i}^{s}=M_{s 01}^{s}=s !\left(\prod_{j=1}^{s} A_{j}\right), \text { for } s \geq 1,
\end{aligned}
$$

where $A_{j}=\beta_{j 1} /\left(\beta_{j 1}+\gamma\right), B_{j}=\gamma /\left(\beta_{j 1}+\gamma\right)$ and the natural definition $\prod_{j=s+1}^{s} A_{j} \equiv 1$.

Remark 4 Expressions (9) to (13) are also valid for models with exposed periods described through a general latency rate function: $\sigma(e) \equiv \sigma_{e}$.

\section{$5 \quad$ Numerical results}

Next we concentrate into show numerical illustrations for the behavior of random variables $R_{e 0}$ and $R_{p}$, assuming that the only infective individ- 
ual in the population is just the patient zero or index case, that is, the starting situation is described by the initial state of the Markov chain $(S(0), E(0), I(0))=(N-1,0,1)$. Let us begin by considering $R_{e 0}$, the number of secondary cases arising from the patient zero while he is infected. Figure 1 presents mass distribution functions of the secondary cases arising in a population of $N=100$ individuals, where the infective process is transmitted according to the mass action transmission, i.e. $\beta_{s i}=\beta s i / N$; contact rate $\beta$ is taken as 10.0 , the exponential infective period has a rate $\gamma=1.0$, so individuals are infected, in mean terms, during a unit time (u.t.). We display four graphs corresponding to four choices of the latency rate $\sigma=0.05,0.5,1.0$ and 10.0, their markers indicate the value of the probabilities $x_{N-1,0,1}^{n}$ for $n$ secondary cases, lines are depicted in order to distinguish among considered situations.

Notice that the probability of having no secondary cases arising from the index case does not depend on $\sigma$ and is given by

$$
\begin{aligned}
x_{N-1,0,1}^{0} & =P\left[R_{e 0}=0 \mid(S(0), E(0), I(0))=(N-1,0,1)\right] \\
& =\gamma /(\gamma+\beta+\beta / N)=0.09174 .
\end{aligned}
$$

For $\sigma=0.05,0.5$ and 1.0 distributions present a decreasing shape, while for $\sigma=10.0$ (i.e., the shortest mean exposed period) mass function presents a bimodal shape with a first mode at $n=0$ and the second one at $n=6$. Although exposed individuals do not transmit the epidemics, infections with large latency periods (that is, small values for $\sigma$ ) give more chance to a big number of secondary infections than infective processes with short latent periods. For instance the probability of having at least 15 secondary cases arising from the index case, is under 0.0021 when $\sigma=10.0$ and it is over 0.12 for $\sigma=0.05,0.5$ or 1.0, which shows the importance of good estimations on the latency rate. 


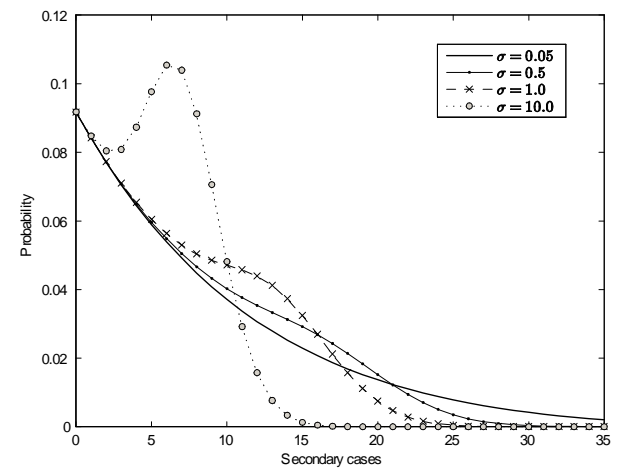

Figure 1: $R_{e 0}$ distribution, mass action incidence function with $\beta=10.0$ and

$$
\gamma=1.0
$$

Figures 2 and 3 are contour graphs corresponding to the expected secondary cases $\bar{R}_{e 0}=E\left[R_{e 0} \mid(S(0), E(0), I(0))=(N-1,0,1)\right]$, when we vary parameters $\beta$ and $\sigma$. Again we consider a population of $N=100$ individuals and a fixed recovery rate $\gamma=1.0$, so we take the mean length of the infective period as the unit time. The incidence function $\beta_{s i}$ is the bilinear mass action for Figure 2 and in Figure 3 is a rescaled version of the incidence function in Xiao \& Ruan [12] modeling psychological effect from the behavioral change of susceptible individuals when the number of infective is getting large. In more details we consider $\beta_{s i}=\beta$ si $/ N$ and $\beta_{s i}=\beta s i /\left(N+4 i^{2}\right)$, respectively; incidence rates share the same infection force $\beta i$ but the nonlinear case describes an inhibitory effect for susceptible individuals which produces a decrease in the number of contacts between susceptible and infective, when number of infective is larger than 5 individuals.

In contrast to deterministic SEIR model, we observe that expected number of secondary cases varies with latency parameter. In more detail, both figures show that $\bar{R}_{e 0}$ decreases with $\sigma$ and this decreasing is more noticeable for large values of $\beta$, while they show an increasing behavior on the expected amount of secondary cases when the proportionality parameter $\beta$ increases. This result is intuitively true, large values of $\beta$ imply large infection force and small values of $\sigma$ provide large latency periods that contribute to increase the expansion of the epidemic process. We can also appreciate from the figures the inhibitory effect that conducts to smaller values of ex- 
pected secondary cases when we deal with the nonlinear transmission function. Moreover, both transmission functions conduct to epidemic models with basic reproduction number $R_{0}=\beta / \gamma$ so Figures 2 and 3 give evidences of the overestimating potential of the infection when measured through $R_{0}$, that also was pointed in Artalejo \& Lopez-Herrero [21]. This fact also can be observed from Figure 4 that displays, for an infective process transmitted according to the mass action incidence rate, the expected number of secondary cases coming from the zero patient, $\bar{R}_{e 0}$, the expected number of infective individuals arising from all infected individuals until the first recovery, $\bar{R}_{p}=E\left[R_{p} \mid(S(0), E(0), I(0))=(N-1,0,1)\right]$, and the basic reproduction number $R_{0}$ when contact rate parameter $\beta$ varies in $(0,30.0)$. As in previous examples, recovery rate is taken as $\gamma=1.0$.

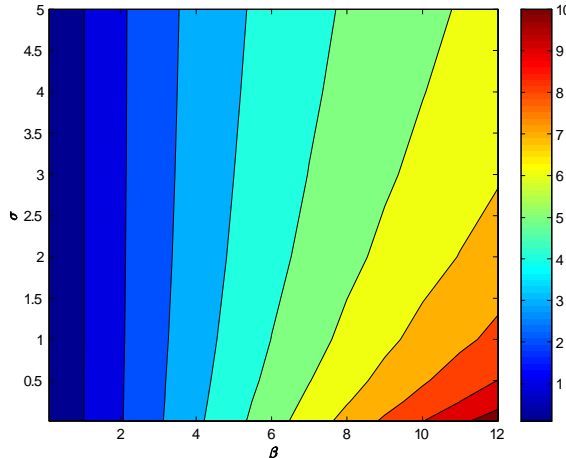

Figure 2: $\bar{R}_{e 0}$ with mass action rate

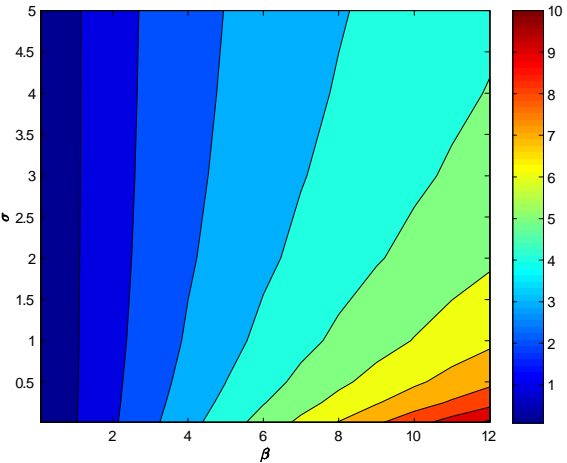

Figure 3: $\bar{R}_{e 0}$ with nonlinear incidence rate

Several curves are drawn by varying latency rate and population size. Due to our choice on recovery rate, the first quadrant bisector corresponds to $R_{0}$ that is an upper bound for $\bar{R}_{e 0}$ and $\bar{R}_{p}$, as functions of $\beta$. Consequently, for moderately sized populations the basic reproduction number counts not only the cases arising from patient zero but even more than these cases coming from all the infective individuals since the origin of the epidemic process and until the first recovery occurs. 


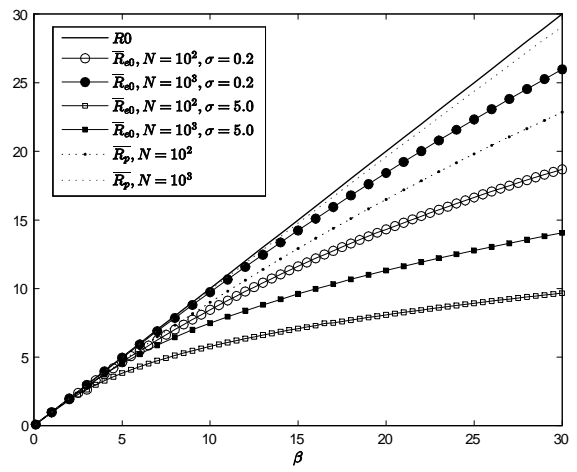

Figure 4: $R_{0}, \bar{R}_{e 0}$ and $\bar{R}_{p}$ as functions of $\beta$

When population size increases, differences between $R_{0}$ and $\bar{R}_{e 0}$ or $R_{0}$ and $\bar{R}_{p}$, become smaller. On the other side, $\bar{R}_{e 0}$ shows a decreasing behavior which is in concordance with the observation made for mass functions after Figure 1. Finally, as the mass action incidence function satisfies the condition stated on Remark 3, the expected number of secondary cases produced by all the infective individuals before the first recovery, $\bar{R}_{p}$, does not depend on $\sigma$.

Figure 5 displays mass distribution function $y_{N-1,0,1}^{n}, 0 \leq n \leq N-1$, of $R_{p}$ for a population of $N=100$ individuals, where recovery rate is $\gamma=1.0$ and we choose latency parameter $\sigma$ in $\{0.05,0.5,1.0,10.0\}$. The infective process is transmitted according to a nonlinear incidence rate of Xiao \& Ruan type [12], that is $\beta_{s i}=\beta s i /\left(N+\alpha i^{2}\right)$, with $\beta=1000.0$ and $\alpha=400.0$, that represents a panic scenario in which, as a reaction of the high level of the force of the infection, the psychological or inhibitory effect appears almost 
immediately in the susceptible individuals.

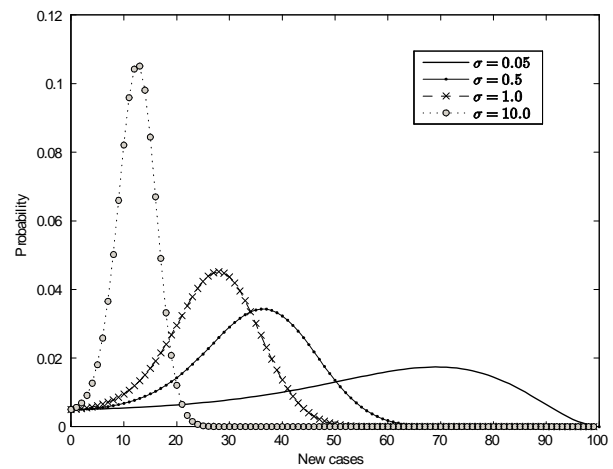

Figure 5: $R_{p}$ under nonlinear transmission in a panic scenario

As in Figure 1, markers correspond to probability values, lines are added for a better distinction among the studied situations. In every case the probability of having no secondary cases is $y_{99,0,1}^{0}=\gamma /\left(\beta_{99,1}+\gamma\right)=0.00502$. All mass functions show a peak, or mode, which is more pronounced for large values of $\sigma$. Moreover, the number of arising secondary cases are more concentrated around the mode for large latency rates, while for small values of $\sigma$ distributions are sparse and present larger modes.

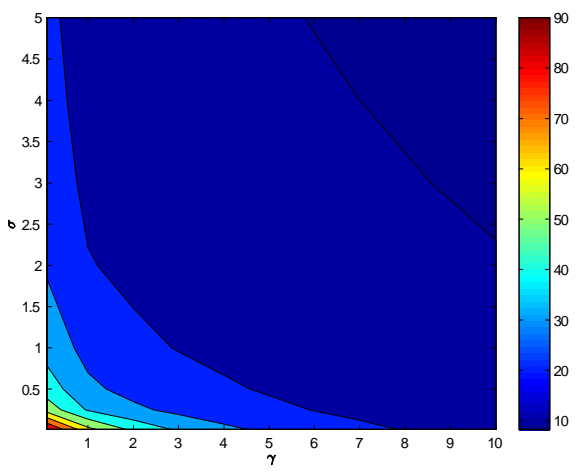

Figure $6: \bar{R}_{p}$ in a panic scenario

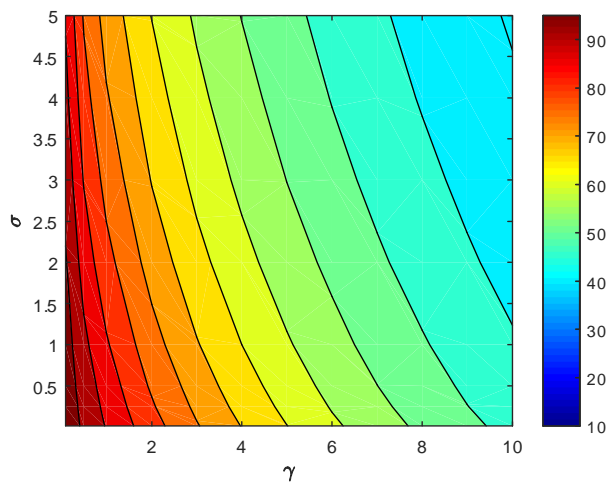

Figure 7: $\bar{R}_{p}$ under slow response 
Finally, Figures 6 and 7 are contour maps of $\bar{R}_{p}$, when varying recovery and latency rates. The incidence transmission function is again of Xiao \& Ruan type with proportionality constant $\beta=1000.0$, parameter measuring inhibitory effect is 400.0 and 4.0, respectively. First choice corresponds the above described panic scenario and second one corresponds to a situation with slower inhibition response, in fact behavioral changes show when the number of infective individuals exceeds 5 .

Both pictures show that $\bar{R}_{p}$ is decreasing as a function of $\sigma$ and also as a function of $\gamma$. Evidencing again that secondary cases increase with increasing recovery and/or latency periods. Notice that large recovery times give chance to a large contacts among individuals. On the other side large latency periods contribute to hide the real spread of the infection. By comparison between both figures we notice that a delayed reaction produces large expected number of cases of infection, on the contrary in a panic situation the inhibitory effect curbs the expansion of the epidemic and produce less contagion cases.

\section{Discussion and concluding remarks}

In this paper we quantify the evolution, in a closed population, of an infective disease with latency period and a finite nonlinear incidence rate.

Main purpose of this work is to provide random measures of the spread of the epidemics, namely $R_{e 0}$ and $R_{p}$, alternative to the basic reproduction number. We present algorithmic procedures for characterizing their distributions.

When dealing with an incidence function satisfying $\beta_{s i}=i * \beta_{s 1}, R_{p}$ generating function, mass function and moments can be determined from explicit expressions which are independent of $\sigma$. Numerical results evidence that $R_{e 0}$ and $R_{p}$ (except for the above mentioned situation) are influenced by the latency period.

A possible extension of this paper could be to study the epidemic expansion when control measures, such as vaccination or isolation, are implemented. Another possibility is to apply the BSDE approach [27] to the study the spread of infections for non-homogeneous Markov-modulated models, because the BSDE approach produces efficient computation of system's performance and helps to reduce the computational costs derived from the large size of the matrices involved in models based on Markovian arrival processes 


\section{Acknowledgments}

The author is grateful to the two anonymous referees for their constructive comments which were really helpful to improve the original manuscript. This work was supported by the Government of Spain (Department of Science, Technology and Innovation) and the European Commission through project MTM 2014-58091.

\section{References}

[1] Kermack WO, McKendrick AG. A contribution to the mathematical theory of epidemics. Proceeding of the Royal Society of London A. 1927; 115: $700-721$.

[2] Anderson RM, May RM. Infectious diseases of humans: dynamics and control. Oxford University Press, Oxford; 1991.

[3] Chowell G, Viboud C, Hyman JM, Simonsen L. The Western Africa ebola virus disease epidemics exhibits both global exponential and local polinomial growh rates. PLOS Current Outbreaks 2015; doi: 10.1371/currents.outbreaks.8b55f4bad99ac5c5db3663e916803261

[4] Viboud C, Simonsen L, Chowell G. A generalized-growth model to characterize the early ascending phase of infectious disease outbreaks. Epidemics. 2016; 15: 27-37.

[5] Severo NC. Generalizations of some stochastic epidemic models. Mathematical Biosciences. 1969; 4: 395-402.

[6] Liu WM, Hethcote HW, Levin SA. Dynamical behavior of epidemiological models with nonlinear incidence rates. Journal of Mathematical Biology. 1987; 25: 359-380.

[7] Capone F, De Cataldis V, De Luca R. On the nonlinear stability of an epidemic SEIR reaction-diffusion model. Ricerche di Matematica. 2013; 62: 161-181.

[8] Korobeinikov A, Maini PK. Non-linear incidence and stability of infectious disease models. Mathematical Medicine and Biology. 2005; 22: 113-128. 
[9] Zhang XB, Huo HF, Xiang H, Meng XY. Dynamics of the deterministic and stochastic SIQS epidemic model with non-linear incidence. Applied Mathematics and Computation. 2014; 243: 546-558.

[10] Lahrouz A, Omari L. Extinction and stationary distribution of a stochastic SIRS epidemic model with non-linear incidence. Statististics $\&$ Probability Letters. 2013; 83: 960-968.

[11] Capasso V, Serio G. A generalization of the Kermack-McKendrick deterministic epidemic model. Mathematical Biosciences. 1978; 42: 41-61.

[12] Xiao D, Ruan S. Global analysis of an epidemic model with nonmonotone incidence rate. Mathematical Biosciences. 2007; 208: 419429.

[13] Li D, Cui J, Liu M, Liu S. The evolutionary dynamics of stochastic epidemic models with nonlinear incidence rate. Bulletin of Mathematical Biology. 2015; 77: 1705-1743.

[14] Cui J, Sun Y, Zhu H. The impact of media on the control of infectious diseases. Journal on Dynamics and Differential Equations. 2008; 20: 31-53.

[15] Cui J, Tao X, Zhu H. An SIS infection model incorporating media coverage. Rocky Mountain Journal of Mathematics. 2008; 38: 13231334 .

[16] Hethcote HW, Levin SA. Periodicity in epidemiological models. In: Gross L, Hallam TG, Levin S A (eds.). Applied Mathematical Ecology; 1989: 193-211.

[17] Alexander ME, Moghadas SM. Bifurcation analysis of an SIRS epidemic model with generalized incidence. SIAM Journal on Applied Mathematics. 2005; 65: 1794-1816.

[18] Turnes PP Jr, Monteiro LHA. An epidemic model to evaluate the homogeneous mixing assumption. Communications in Nonlinear Science and Numerical Simulation. 2014; 19: 4042-4047.

[19] Heesterbeek JAP, Dietz K. The concept of $R_{0}$ in epidemic theory. Statistica Neerlandica. 1996; 50: 89-110.

[20] Hethcote HW. The mathematics of infectious diseases. SIAM Review. 2000; 42: 599-653. 
[21] Artalejo JR, Lopez-Herrero MJ. On the exact measure of disease spread in stochastic epidemic models. Bulletin of Mathematical Biology. 2013; 75: 1031-1050.

[22] van den Driessche P, Watmough J. Reproduction numbers and subthreshold endemic equilibria for compartmental models of disease transmission. Mathematical Biosciences. 2002; 180: 29-48.

[23] Britton T. Stochastic epidemic models: a survey. Mathematical Biosciences. 2000; 225: 24-35.

[24] Artalejo JR, Economou A, Lopez-Herrero MJ. The stochastic SEIR model before extinction: Computational approaches. Applied Mathematics and Computation. 2015; 265: 1026-1043.

[25] Ross JV. Invasion of infectious diseases in finite homogeneous populations. Journal of Theoretical Biology. 2011; 289: 83-89.

[26] Abate J, Whitt W. Numerical inversion of Laplace transforms of probability distributions. ORSA Journal on Computing. 1995; 7: 36-43.

[27] Artalejo JR, Gómez-Corral A. A state-dependent Markov-modulated mechanism for generating events and stochastic models. Mathematical Methods in the Applied Sciences. 2010; 33: 1342-1349. 


\section{FIGURES}

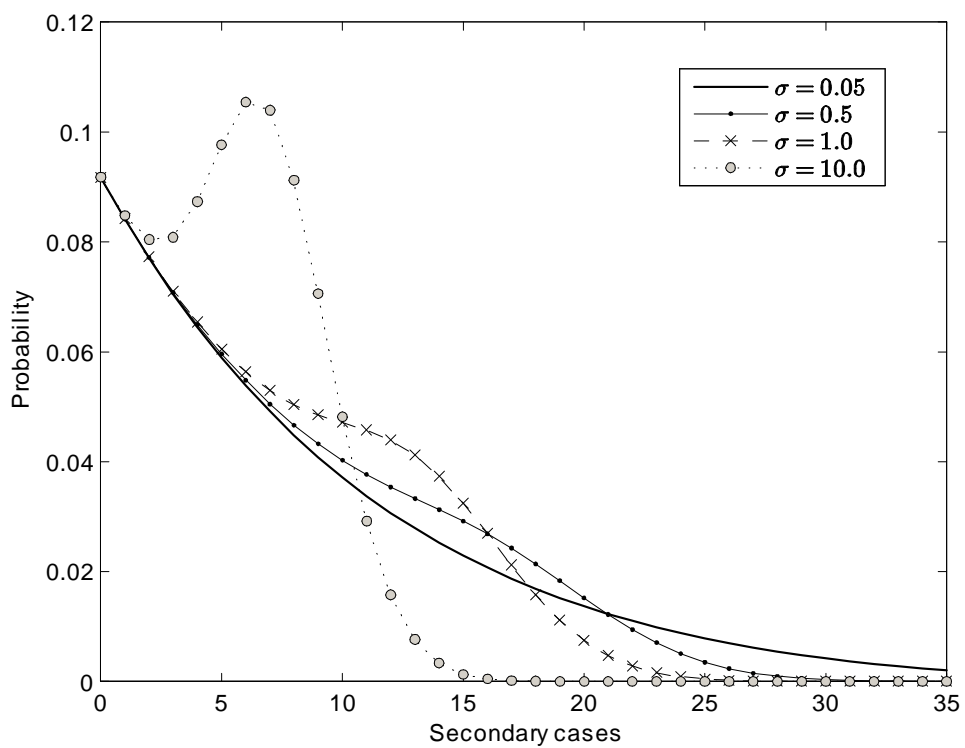

Figure 1: $R_{e 0}$ distribution, mass action incidence function with $\beta=10.0$ and $\gamma=1.0$ 


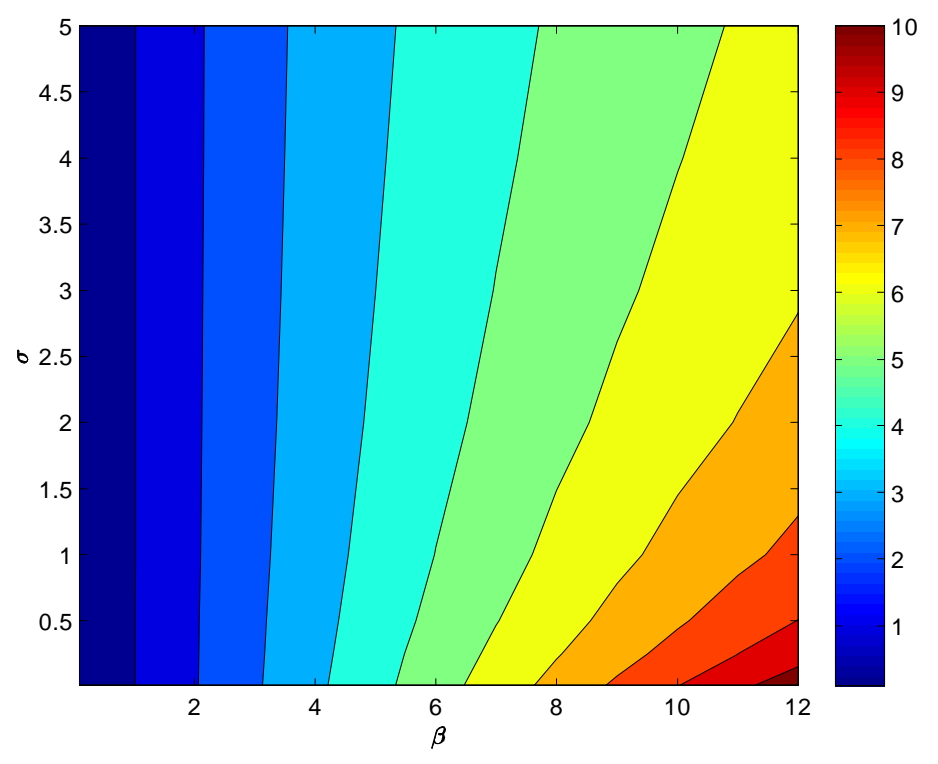

Figure 2: $\bar{R}_{e 0}$ with mass action rate 


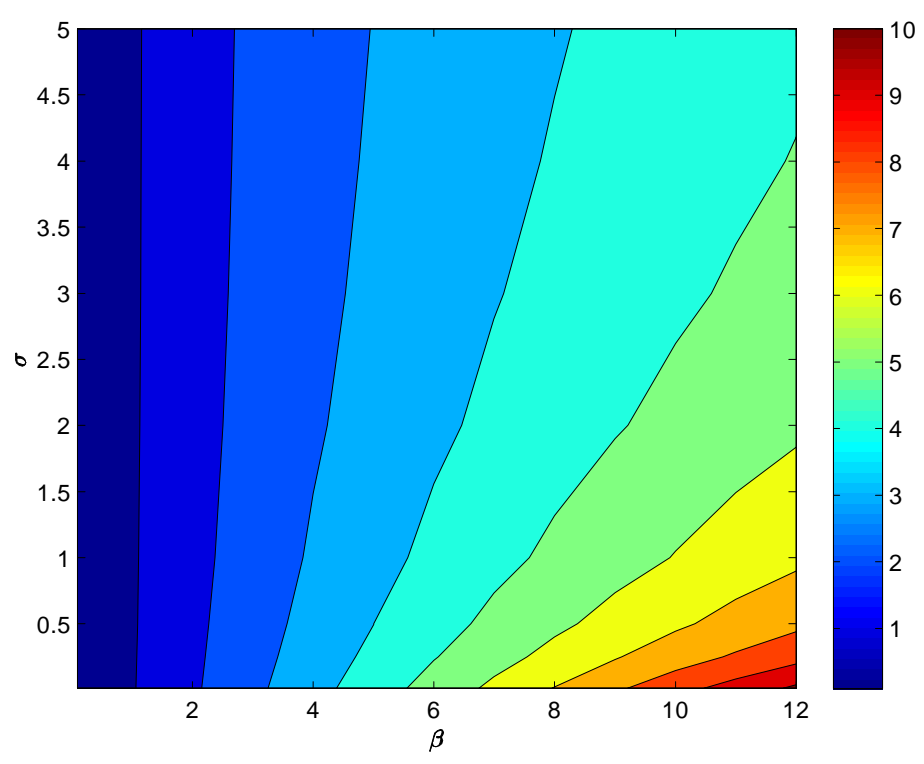

Figure 3: $\bar{R}_{e 0}$ with nonlinear incidence rate 


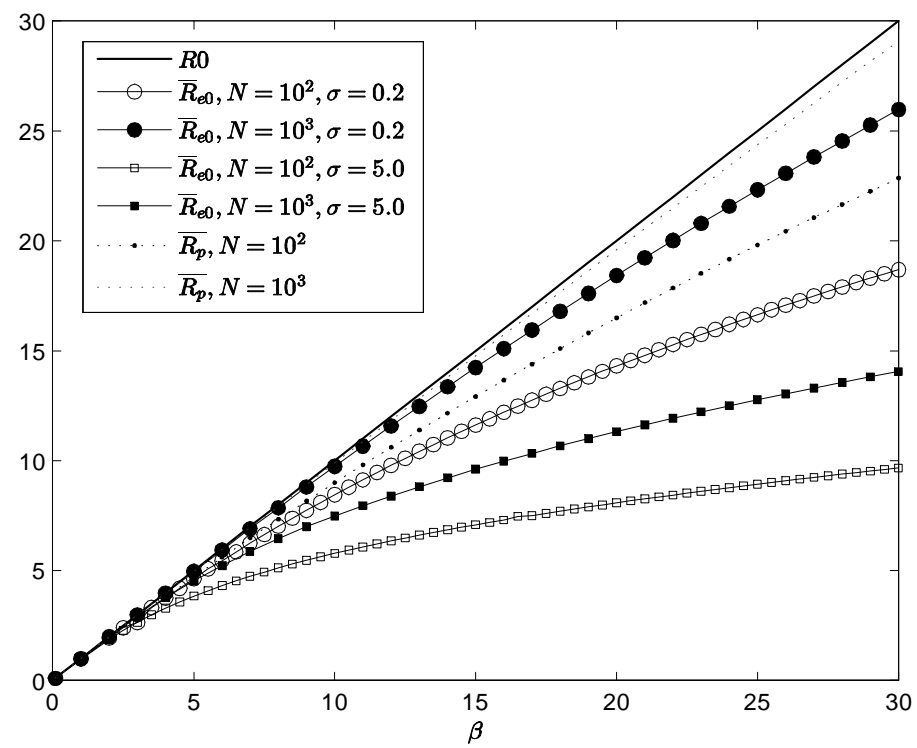

Figure $4: R_{0}, \bar{R}_{e 0}$ and $\bar{R}_{p}$ as functions of $\beta$ 


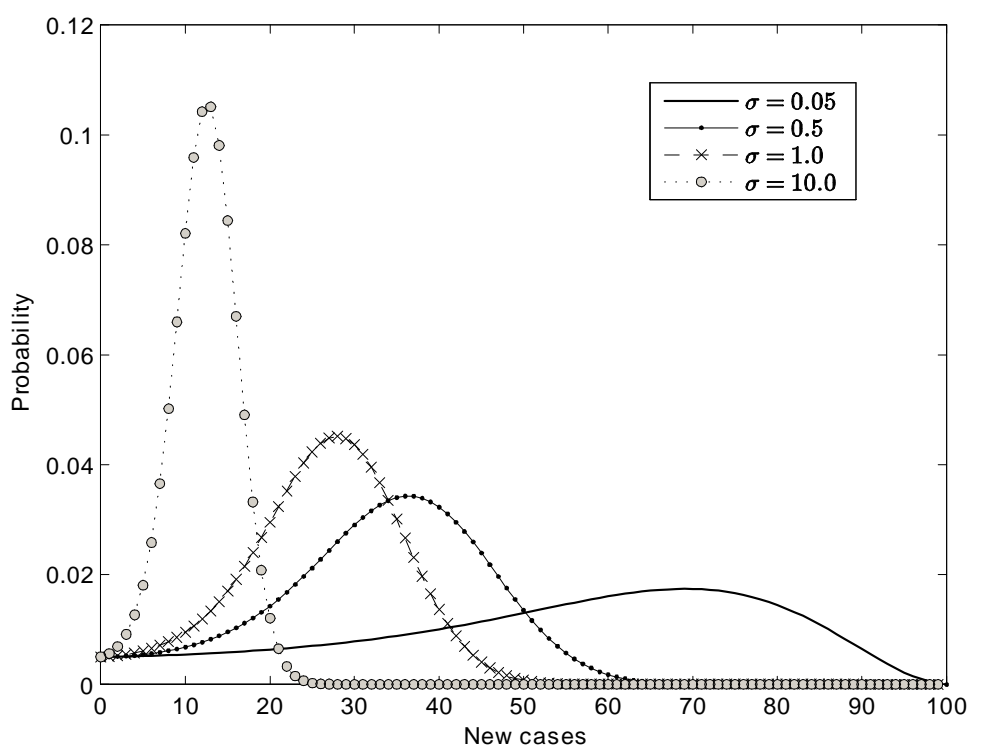

Figure 5: $R_{p}$ under nonlinear transmission in a panic scenario 


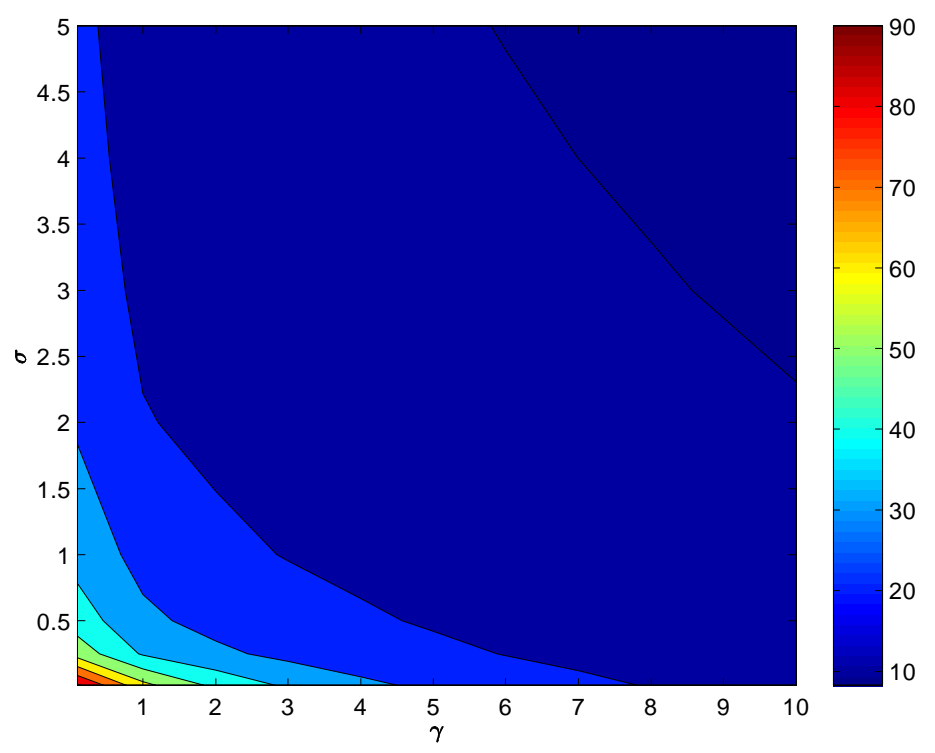

Figure 6: $\bar{R}_{p}$ in a panic scenario 


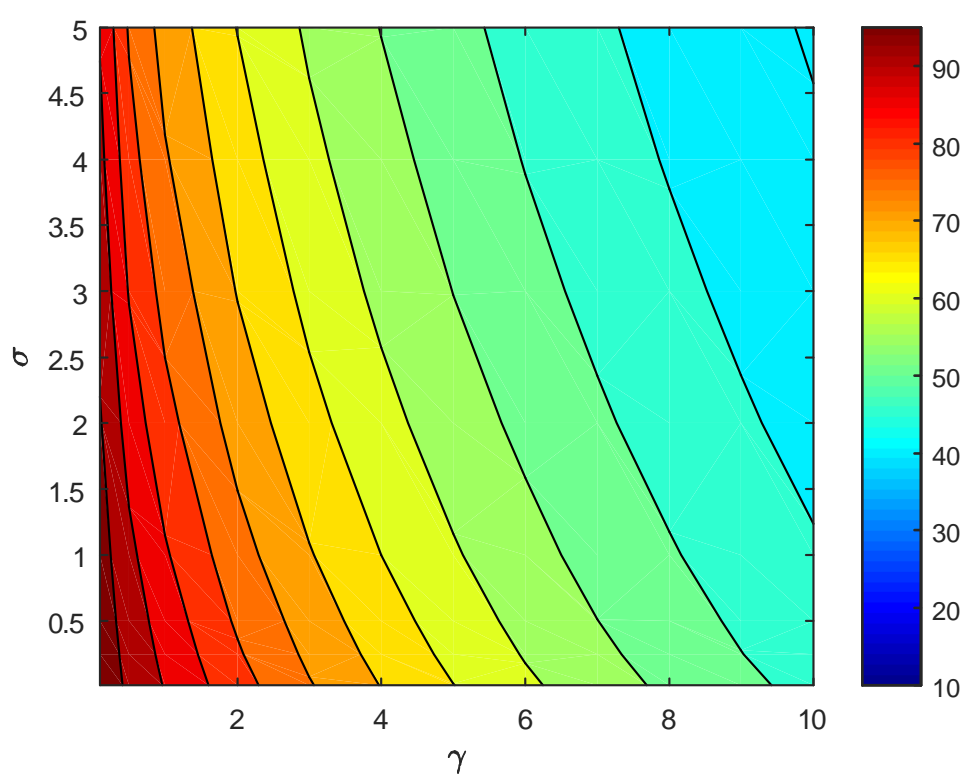

Figure $7: \bar{R}_{p}$ under slow response 